\title{
Basal and Luminal Molecular Subtypes in Naturally-Occurring Canine Urothelial Carcinoma are Associated with Tumor Immune Signatures and Dog Breed
}

\author{
Breann C. Sommer ${ }^{\mathrm{a}, 1,2}$, Deepika Dhawan ${ }^{\mathrm{a}, 2}$, Audrey Ruple ${ }^{\mathrm{b}, \mathrm{c}}$, José A. Ramos-Vara ${ }^{\mathrm{c}, \mathrm{d}}$, \\ Noah M. Hahn ${ }^{\text {e }}$, Sagar M. Utturkar ${ }^{c}$, Elaine A. Ostrander ${ }^{\mathrm{f}}$, Heidi G. Parker ${ }^{\mathrm{f}}$, \\ Christopher M. Fulkerson $^{\mathrm{a}, \mathrm{c}}$, Michael O. Childress ${ }^{\mathrm{a}, \mathrm{c}}$, , Lindsey M. Fourez ${ }^{\mathrm{a}}$, \\ Alexander W. Enstrom ${ }^{\mathrm{a}}$ and Deborah W. Knapp ${ }^{\mathrm{a}, \mathrm{c}, *}$ \\ ${ }^{a}$ Department of Veterinary Clinical Sciences, Purdue University, West Lafayette, IN, USA \\ ${ }^{\mathrm{b}}$ Department of Public Health, Purdue University, West Lafayette, IN, USA \\ ${ }^{\mathrm{c}}$ Purdue University Center for Cancer Research, West Lafayette, IN, USA \\ ${ }^{\mathrm{d}}$ Department of Comparative Pathobiology, Purdue University, West Lafayette IN, USA \\ ${ }^{\mathrm{e}}$ Department of Oncology and Urology, and Sidney Kimmel Comprehensive Cancer Center, Johns Hopkins \\ University, Baltimore, MD, USA \\ ${ }^{\mathrm{f}}$ National Human Genome Research Institute, National Institutes of Health, Bethesda, MD, USA
}

Received 3 December 2020

Accepted 19 May 2021

Pre-press 4 June 2021

Published 31 August 2021

\begin{abstract}
.
BACKGROUND: Improved therapies are needed for patients with invasive urothelial carcinoma (InvUC). Tailoring treatment to molecular subtypes holds promise, but requires further study, including studies in pre-clinical animal models. Naturallyoccurring canine InvUC harbors luminal and basal subtypes, mimicking those observed in humans, and could offer a relevant model for the disease in people.

OBJECTIVE: To further validate the canine InvUC model, clinical and tumor characteristics associated with luminal and basal subtypes in dogs were determined, with comparison to findings from humans.

METHODS: RNA sequencing (RNA-seq) analyses were performed on 56 canine InvUC tissues and bladder mucosa from four normal dogs. Data were aligned to CanFam 3.1, and differentially expressed genes identified. Data were interrogated with panels of genes defining luminal and basal subtypes, immune signatures, and other tumor features. Subject and tumor characteristics, and outcome data were obtained from medical records.
\end{abstract}

\footnotetext{
${ }^{1}$ Dr. Sommer's current address is: VCA Veterinary Emergency Service \& Veterinary Specialty Center, Madison, WI, USA.

${ }^{2}$ Drs. Sommer and Dhawan contributed equally to this work and as such are co-first authors.
}

\footnotetext{
${ }^{*}$ Correspondence to: Deborah W. Knapp, Department of Veterinary Clinical Sciences, Purdue University, 625 Harrison St., West Lafayette, IN 47907-2026, USA. Tel.: +1 765494 9900; Fax: +1 765496 1108; E-mail: knappd@purdue.edu.
} 
RESULTS: Twenty-nine tumors were classified as luminal and 27 tumors as basal subtype. Basal tumors were strongly associated with immune infiltration (OR 52.22, 95\% CI 4.68-582.38, $P=0.001$ ) and cancer progression signatures in RNAseq analyses, more advanced clinical stage, and earlier onset of distant metastases in exploratory analyses $(P=0.0113)$. Luminal tumors were strongly associated with breeds at high risk for InvUC (OR 0.06, 95\% CI $0.01-0.37, P=0.002$ ), non-immune infiltrative signatures, and less advanced clinical stage.

CONCLUSIONS: Dogs with InvUC could provide a valuable model for testing new treatment strategies in the context of molecular subtype and immune status, and the search for germline variants impacting InvUC onset and subtype.

Keywords: Muscle invasive bladder cancer, animal model, subtype, T-cell-inflamed, breed, RNA-seq

\section{INTRODUCTION}

Urinary bladder cancer is newly diagnosed in more than 80,000 people each year in the United States, with 18,000 deaths expected in 2020 [1]. Muscle invasive bladder cancer, specifically invasive urothelial carcinoma (InvUC) is a particularly aggressive form of bladder cancer, and is lethal in approximately half of patients [2]. The treatment of InvUC has typically involved cystectomy and platinum-based chemotherapy, with five-year survival rates ranging from 5 $-79 \%$ depending on the stage at diagnosis [3]. The management of InvUC, however, is rapidly evolving [3-5]. Multiple immune checkpoint inhibitors have been approved for use in patients with InvUC, and these drugs are inducing durable remission of highly advanced cancer, albeit in small subsets of patients [3-6]. Another recent advancement has been the identification of tumor RNA expression patterns that segregate InvUC into molecular subtypes, with cancer behavior and treatment responses differing between subtypes [7-11]. There is strong interest in developing strategies that apply subtype information to build predictors of patient outcomes and to select optimal therapies in individual patients.

The molecular subtypes of InvUC are defined at the highest level as luminal and nonluminal, with subgroupings within each subtype [7-13]. Subgroups within the nonluminal group can include basal/squamous, small cell / neuroendocrine, and stroma-rich tumors (mesenchymal like) [12]. Within luminal tumors, urothelial and genomically unstable subgroups have been described. In initial studies of molecular subtypes and in studies in which the tumors have been divided into only two subtypes, nonluminal tumors are often referred to as basal subtype tumors [8, 12].

Women with InvUC have a higher prevalence of basal subtype cancer, which is inherently more aggressive. Basal InvUC is often associated with advanced stage and metastatic disease at presentation as well as shorter overall survival $[10,11]$. In contrast, luminal InvUC appears to be inherently less aggressive, and is enriched with FGFR 3 mutations that can be targeted for therapy $[10,11]$. Patients with basal tumors appear to receive more clinical benefit from platinum-based neoadjuvant chemotherapy compared to patients with luminal tumors [9-11, 15]. Finally, basal InvUC is also characteristically more immune infiltrated [9-11, 15-17], and is typically more responsive to immunotherapies than luminal tumors $[10,14]$.

Multiple new therapeutic strategies including combination therapies in the context of molecular subtypes are being developed. The numbers of patients eligible for clinical trials, however, is insufficient to complete even a portion of the trials needed to test these strategies. This underscores the importance and need for studies in relevant pre-clinical animal models to select promising strategies to move to humans. Experimental animal models utilized in well-controlled studies are clearly essential in advancing bladder cancer research, however, these models often lack many of the complexities of human cancer $[18,19]$. As a complementary model approach, dogs develop naturally-occurring InvUC which mimics human InvUC in clinical signs, cellular and pathological features of disease including high grade, tumor heterogeneity, local invasion, distant metastases, response to chemotherapy, and important for this discussion, luminal and basal molecular subtypes [20-22]. Interestingly, the development of InvUC in dogs is strongly associated with the breed of the dog. Compared to mixed breed dogs, Scottish Terriers have a 20-fold increased risk for InvUC, and West Highland White Terriers, Shetland Sheepdogs, Wire Hair Fox Terriers and Beagles have a three- to sixfold increased risk for the cancer [22, 23]. Another intriguing finding is that the majority of cases harbor a $B R A F^{V 595 E}$ mutation, the canine homologue of $B R A F^{V 600 E}$ that is a common driver of many types of human cancer $[24,25]$. Although $B R A F$ mutations are uncommon in human bladder cancer, such mutations have been noted in patients with especially aggressive bladder cancer [7, 26]. Interestingly, while 
$B R A F$ mutations are common in canine InvUC and uncommon in human InvUC, in both species similar molecular subtypes are observed [21].

While the presence of luminal and basal subtypes have been described in an initial group of dogs [21], the clinical and pathological features associated with the subtypes have not been reported. This information would be important in validating the canine model. The purpose of this study, therefore, was to determine clinical features and tumor characteristics associated with luminal and basal subtypes in canine InvUC, and to compare findings to those reported in humans. The study demonstrates multiple instances in which the clinical features and tumor characteristics associated with subtype in dogs are consistent with those reported in humans. The association between immune signatures and subtype was especially compelling. The study also revealed a strong association between subtype and dog breed which could be exploited in future studies to expand the understanding of genomic drivers of InvUC.

\section{MATERIAL AND METHODS}

\section{Canine tissue samples}

All tissues from dogs were collected with the approval of the Purdue Animal Care and Use Committee (protocol approval \#1111000169). Canine InvUC tissues were collected by cystoscopic biopsy, surgical biopsy, or necropsy of dogs presenting to the Purdue University Veterinary Teaching Hospital for evaluation and treatment of bladder cancer. The samples were stored in trizol (Invitrogen, Carlsbad, CA) at $-80^{\circ} \mathrm{C}$, and tissues with histopathologic confirmation of InvUC were processed for RNA isolation. Of the 56 cases included in this study, the RNA-sequencing (RNA-seq) data from 29 tumors has been published previously [21]. Normal canine bladder tissue samples $(n=4)$ were collected from dogs that were being euthanized for conditions other than bladder-related diseases. The urothelial layer was stripped from full thickness bladder using fine dissection and stored immediately in trizol at $-80^{\circ} \mathrm{C}$ for subsequent RNA isolation.

\section{RNA-seq of canine InvUC tumor samples and normal canine bladder mucosa}

Total RNA was isolated following manufacturer's instructions (Invitrogen, Carlsbad, CA) and purified using RNAeasy (Qiagen, Valencia, CA). The samples were processed for RNA-seq and sequenced at the Biomedical Genomics Core at Nationwide Children's Hospital, Columbus, Ohio. The quantity and quality of total RNA was evaluated using NanoDrop spectrophotometer (NanoDrop Technologies, Wilmington, DE) and Agilent Bioanalyzer (Agilent Technologies, Santa Clara, CA). RNA quality was considered acceptable if the RNA integrity number was seven or greater. Ribosomal-RNA was removed from total RNA with Ribo-ZeroTM rRNA removal kit (Illumina), and mRNA libraries were constructed (ScriptSeqTM v2 RNA-Seq library preparation kit, Epicentre Biotech, Madison, WI). Di-tagged cDNA was amplified by limit-cycle PCR and purified using AMPure XP System (Beckman Coulter) [21, 27]. Paired-end 150 bp sequence reads were generated using Illumina HiSeq 4000 platform to obtain an average of 50 million reads/sample. Raw reads were cleaned for PCR artifacts and adapter trimmed, and then aligned to the CanFam 3.1 reference genome. COBWeb was used to obtain expression levels for annotated genes and isoforms (Strand NGS, v3.1, Build 235027, Agilent Technologies). Analyses were conducted using genes published to be of importance in human InvUC described below, and the canine orthologs were mapped and used for analyses. The orthologous genes were mapped using ensembl biomart service in combination with Strand NGS. Non-orthologous genes were not considered.

\section{RNA-seq data analyses}

RNA-seq analyses were performed using Strand NGS (Agilent Technologies). Pairwise comparison between normal and InvUC samples was used to detect differential expression of genes by applying DESEQ2 on DESEQ normalized data and edge $\mathrm{R}$ on TMM normalized data (fold change $\geq 2$, $\mathrm{p}$ corr $<0.05)$ [28, 29] The differentially expressed genes in the RNA-seq data were specifically interrogated for genes used to assign human InvUC into molecular subtypes, e.g. luminal and basal using a previously reported 60-gene prediction panel [21]. This panel was established using Support Vector Machine algorithm, validation parameters with 100,000 iterations, and the validation type "Leave One Out" to generate the validation algorithm outputs as previously described [21].

To confirm the stability of the subtype assignment, consensus clustering was performed using R-package ConsensusClusterPlus (1000 bootstraps, $80 \%$ subsamples, MaxK = 10) using the 60-gene panel and the 
$\log 2 \mathrm{FC}$ values (normal vs. tumor) applying a range from $K=2$ to $K=10$ [30]. The consensus score matrix was visualized as a heatmap.

The data were also interrogated for genes used to characterize the tumor immune status [31], and other gene panels $[17,21,31-33]$ using the canine orthologs. The immune-related genes were those reported to classify human bladder cancer as nonT-cell-inflamed (not infiltrated by $\mathrm{T}$ lymphocytes, immune "cold"), or T-cell-inflamed (infiltrated by T lymphocytes, immune "hot") [31]. The data were visualized in heatmap format using $\mathrm{R}$ package pheatmap. Rows (genes) were grouped by hierarchical clustering based on Euclidean distance and "complete" agglomeration method. A semi-quantitative approach based on the percentage of immune genes expressed in each tumor was used to assign each tumor an "RNA-seq immune score" from 1 to 5 with 1 being the coldest tumor score, and 5 being the hottest tumor score as follows: score of $1=0-15 \%$ of genes, score of $2=16-30 \%$ of genes, score of $3=31-50 \%$ of genes, score of $4=51-70 \%$ of genes, and score of $5=>70 \%$ of genes overexpressed. In additional analyses, the data were interrogated for genes associated with cancer progression across cancer types (nCounter® PanCancer Progression Gene Panel, $n=770$ genes, Nanostring Technologies, Seattle, WA) [34]. Gene ontology analyses were conducted in Strand NGS applying Fisher test with multiple test correction (Bonferroni), and the top 20 GO terms (p corr < 0.001) compiled [35].

SNP detection was performed on RNA-seq data to assign $B R A F^{V 595 E}$ mutation status using Strand NGS. Briefly, dbSNP ensembl was used as annotation for SNP detection. The filters applied for SNP detection were: SB50TR100 (percent strand bias $>50$ and total reads $>100$ ), SRP4 (supporting reads $\%<4$ ), HomPolyFilter in which in homopolymer stretches indels below $25 \%$ supporting reads were removed even with total number of reads $>40$, LowBQ (low base quality where average base quality is $<15$ ), IndelSR20 (indels with supporting reads below 20\%), and TotalReadsFilter (total reads filtered below 20). In addition, reference locations with coverage below 10 and split and partial reads were ignored.

\section{Clinical and pathological data}

The following information was compiled from the dogs' medical records: sex, neuter status, breed, age at diagnosis, tumor location and size, treatments received, TNM stage at diagnosis and death, time of the onset of distant metastases, and overall survival time defined as the time between cancer diagnosis and death from any cause. Tumor stage was assessed by thoracic and abdominal radiography, abdominal ultrasonography with standardized cystosonography, and necropsy when available. The TNM stage was classified following WHO criteria for canine tumors [36]. High-risk breeds for the development of InvUC were defined as Scottish Terrier, West Highland White Terrier, Wire Hair Fox Terrier, Shetland Sheepdog, and Beagle [23, 37]. Non-high risk breeds were defined as other breeds and mixed breed dogs.

All tumor samples were reviewed by a single pathologist (JRV). The InvUC grade was assigned following a four-tier grading system based on criteria described by Cheng et al., with grade 4 being the highest grade [38].

\section{CD3 immunohistochemistry (IHC)}

CD3 IHC (CD3 antibody A0452, Agilent Technologies) was performed to detect tumor infiltrating lymphocytes (TILs) on formalin fixed paraffin-embedded sections as previously described [39, 40]. The percentage of CD3 + cells / 100 tumor cells in the most immune infiltrated area of the section was scored as follows: $1=1-10 \% ; 2=11-25 \% ; 3=26-$ $50 \%$, and $4=>50 \%$ CD3 cells / 100 tumor cells. Intraepithelial and stromal TILs were scored separately.

\section{Sample size and statistical analyses}

The goal was to analyze tumor samples from a minimum of 54 dogs, as this was expected to provide at least 20 dogs each with luminal tumors and basal tumors for correlative clinical study [21]. Logistic regression analysis was performed to assess relationships between subtype and the following variables: age, sex, neuter status, high-risk breed for InvUC, tumor stage, tumor grade, tumor size, tumor location, presence of $B R A F^{V 595 E}$ mutation, RNA-seq immune score, and IHC immune score. Variables with a $P$ value of less than 0.05 in the univariable model were introduced to a multivariable model using stepwise progression. In exploratory analyses, the same variables were analyzed in relation to overall survival time, and the time from diagnosis to development of detectable distant metastases using Cox proportional models. The TNM stage at death and overall survival time were recorded, but were also considered secondary exploratory endpoints, as the dogs were 
not in a specific therapeutic trial, and the treatments varied between dogs. All analyses were performed using StataSE 16 (StataCorp. 2019. Stata Statistical Software: Release 16. College Station, TX: StataCorp LLC.).

\section{RESULTS}

\section{Subject and tumor characteristics}

RNA-seq data from 56 dogs with InvUC were analyzed for this study. Characteristics of the dogs and their tumors are summarized in Table 1. Forty-nine dogs were presented with clinical signs typical of canine InvUC including hematuria, stranguria, and increased frequency of urination. Seven dogs had been participating in a bladder cancer screening study and had no clinical signs of urinary disease. In these dogs, masses were detected by ultrasonography, and subsequent cystoscopic biopsy confirmed InvUC. In subset analyses, tumors in dogs in the screening study were similar to those observed in other dogs with a potential exception related to the immune state discussed below.

Of the 56 tumors, 29 tumors were classified as luminal and 27 tumors as basal subtype (Fig. 1, Fig. 2) with the 60-gene classifier genes listed in Supplementary Table S1. The stability of the subtype assignment was verified by calculating their co-clustering frequency in iterative hierarchical clustering by applying consensus clustering independent of the published class prediction model (Fig. 2). Two distinct clusters containing $90 \%$ of the samples with high $(>0.8)$ consensus score were observed, while approximately $\sim 10 \%$ of the samples were unstable with low $(<0.8)$ consensus score. Consensus clustering was also performed with up to $\mathrm{K}=10$, i.e. forcing the creation of maximum 10 groups, and approximately $60 \%$ of the samples were retained into two distinct groups indicating high stability of the groups.

As with human InvUC, heterogeneity was common within and between samples, and smaller subgroupings within subtypes were observed (Fig. 1). The associations between subtype and clinical and pathologic variables are summarized in Table 2 and discussed below.

\section{Association between dog breed and molecular subtype}

There was a very strong association between breed-risk for InvUC and molecular subtype in the univariable and multivariable analyses (Fig. 1, Table 2). Of $28 \mathrm{dogs}$ in high-risk breeds, $23 \mathrm{dogs}$ $(82.1 \%)$ had luminal tumors, and five dogs (17.9\%) had basal tumors (OR 0.06 for basal tumors, $95 \% \mathrm{CI}$ $0.01-0.37, P=0.002)$. Of $28 \mathrm{dogs}$ in the non-high risk breeds, six dogs $(21.4 \%)$ had luminal tumors, and 22 dogs $(78.6 \%)$ had basal tumors. Luminal tumors were present in 14 of 19 Scottish Terriers, six of six Beagles, two of two West Highland White Terriers, and one of one Shetland Sheepdog.

It was noted that the non-high risk breed group included two Border Collies, and one each of Norfolk Terrier, Airedale Terrier, Wheaten Terrier, Miniature Schnauzer, and Norwegian Elkhound. These breeds are either closely related to the defined high-risk breeds or have been considered as high-risk in some, although not all studies $[22,37]$. The tumors in these seven dogs included four basal tumors and three luminal tumors. When assessing the subtype in all terriers $(n=24$ dogs), 18 dogs $(75.0 \%)$ had luminal tumors, and six dogs $(25.0 \%)$ had basal tumors.

\section{Analyses of other clinical and pathological variables and molecular subtype}

\section{Sex}

Of 27 dogs with basal tumors, 21 dogs $(77.8 \%)$ were female, and six dogs $(22.2 \%)$ were male. Of 29 dogs with luminal tumors, 19 dogs $(65.5 \%)$ were female, and $10 \operatorname{dogs}(34.5 \%)$ were male. Basal tumors comprised $52.2 \%$ of the tumors in females and $37.5 \%$ of the tumors in males. There was not a significant association between sex and subtype (OR 0.54 for male dogs having basal tumors, 95\% CI0.17-1.78, $P=0.30807)$.

\section{Location}

When evaluating any one specific location such as apex, mid-bladder, trigone, urethra, or prostate, there was no association with subtype (Table 2). Two other findings related to location were noted. First, the cancer was present in more than one location in 20 of 29 dogs with luminal tumors $(69.0 \%)$ and in 23 of 27 dogs with basal tumors (85.2\%) (OR 3.60. $95 \%$ CI $0.86-15.12, P=0.064)$. Second, tumors not in the trigone / urethra / prostate, i.e. limited to the apex and mid-bladder, were more often of the luminal subtype. Of $16 \mathrm{dogs}$ with tumors limited to the apex and mid-bladder, five tumors (31.2\%) were basal, and 11 tumors $(68.7 \%)$ were luminal, although the result was not significant. Tumors in the trigone and urethra were comprised of $55.0 \%$ basal tumors and $45.0 \%$ luminal tumors. 
Table 1

Subject and tumor characteristics for the $56 \mathrm{dogs}$ in the study

Characteristic
Age
Sex and neuter status

Findings

Median: 10.4 years (range $6.3-15.0$ years)

Female, spayed: 39 dogs

Female, intact: 1 dog

Male, neutered: 14 dogs

Breed $^{\mathrm{a}}$

Male, intact: 2 dogs

19 Scottish Terriers

11 Mixed breed dogs

6 Beagles

2 West Highland White Terriers

2 Shih Tzus

2 German Shorthair Pointers

2 Border Collies

One each of: Shetland Sheepdog, Norfolk

Terrier, Airedale Terrier, Welsh Springer

Spaniel, Chesapeake Bay Retriever, Bluetick

Hound, Boxer, Wheaten Terrier, Miniature

Schnauzer, Miniature Pinscher, Labrador

Treatments received prior to

Retriever, and Norwegian Elkhound.

tumor tissue collection

None: 38 dogs

Cyclooxygenase inhibitor: $17 \mathrm{dogs}$

Methods of tumor tissue

collection

Chemotherapy: $1 \mathrm{dog}$

Cystoscopy: 47 dogs

Surgery: 7 dogs

Necropsy: $2 \operatorname{dog}^{\mathrm{b}}$

TNM stage at diagnosis according to criteria for CANINE tumors ${ }^{\mathrm{c}}$

T2N0M0:40 dogs

T2N1M0:3 dogs

T2N0M1:2 dogs

T2N1M1:2 dogs

T3N0M0:8 dogs

Incomplete staging information: $1 \mathrm{dog}$

TNM stage at death according to criteria for CANINE tumors, available on $47 \operatorname{dogs}^{c}$

T0N0M0:1 dog

T2N0M0:15 dogs

T2N1M0:4 dogs

T2N0M1:4 dogs

T2N1M1:5 dogs

T3N0M0:4 dogs

T3N1M0:1 dog

T3N0M1:2 dog

T3N1M1:6 dogs

Partial staging information:

T2N1MNA: 2 dogs

T2NNAM0:1 dog

T3N1MNA: $1 \mathrm{dog}$

T3NNAMNA: 1 dog

Tumor location

Bladder trigone: $36 \mathrm{dogs}$

Urethra: 31 dogs

Mid bladder: 28 dogs

Bladder apex: 20 dogs

Prostate: 6 dogs

Tumor in more than one site: 43 dogs

Cancer grade $^{\mathrm{d}}$

Grade 2:3 dogs

Grade 3:12 dogs

Grade 4:39 dogs

Not available: 2 dogs

Molecular subtype

Luminal: 29 dogs

Basal: 27 dogs

Urine $B R A F^{V 595 E}$ mutation

Mutation present: 42 dogs

$B R A F$ wild type: $14 \operatorname{dogs}$ 
Table 1

(Continued)

\begin{tabular}{ll}
\hline Characteristic & Findings \\
\hline Immune score - RNA-seq & $1: 11 \operatorname{dogs}$ \\
& $2: 15 \operatorname{dogs}$ \\
& $3: 11 \operatorname{dogs}$ \\
& $4: 9 \operatorname{dogs}$ \\
& $5: 10 \operatorname{dogs}$ \\
\hline
\end{tabular}

${ }^{a}$ Breeds at high-risk for InvUC were defined as Scottish Terriers, West Highland White Terriers, Shetland Sheepdogs, Wirehair Fox Terriers, and Beagles [22, 23]. ${ }^{\mathrm{b}}$ The owners of two dogs requested euthanasia of their dog following diagnosis, and did not pursue further evaluation and treatment. One of these dogs had received deracoxib. The other dog was untreated. ${ }^{\mathrm{c}}$ The TNM classification system for canine InvUC differs from the classification system for human bladder tumors. In dogs, T2 tumors are invasive into the bladder wall, and T3 tumors extend through the bladder wall to the outside of the bladder or invade adjacent organs [36]. These would be classified at T3 and T4, respectively, in human bladder tumor classification schemes. ${ }^{\mathrm{d}}$ Cancer grade was assigned following criteria described by Cheng et al. [38].

\section{Tumor stage and grade}

At diagnosis, six of ten dogs $(60.0 \%)$ with tumors extending through the serosa to the outside of the bladder or with spread to adjacent organs (T3 in canine tumor staging system), had basal tumors, and four dogs $(40.0 \%)$ had luminal tumors. Of 46 tumors invading into the bladder wall, but not penetrating through the serosa (T2 in canine tumor staging system), 21 tumors $(45.7 \%)$ were basal, and 25 tumors $(54.3 \%)$ were luminal. The $\mathrm{N}$ stage did not differ between luminal and basal tumors (Table 2). Of four dogs with distant metastases (stage M1) at diagnosis, three dogs (75.0\%) had basal tumors, and one dog $(25 \%)$ had a luminal tumor.

The TNM stage at death was an exploratory endpoint. Although the monitoring and staging procedures were similar across cases, and no dogs underwent cystectomy, and the general type of medical therapies were similar between dogs, the specific drug protocol varied between cases. The $T$ stage and $\mathrm{N}$ stage at death did not differ between dogs with basal or luminal tumors. Distant metastases (M1) were present in 17 of 43 dogs with staging information available at death. At the time of death, distant metastases were present in eight of 26 dogs (30.8\%) with luminal tumors, and nine of $17(52.9 \%)$ dogs with basal tumors (OR 2.53, 95\% CI 0.71 - 8.97, $P=0.1465$ ). The median survival was 349 days for all dogs, and did not differ between dogs with luminal vs basal tumors. In other exploratory analyses, the median time between diagnosis and the detection of distant metastasis was significantly shorter in nine dogs with basal tumors (median 110 days, range 0 - 356 days) than in eight dogs with luminal tumors (median 394 days, range $0-1752$ days) $(P=0.0113)$ (Supplementary Figure S1).
The aggressive nature of the basal tumors was also demonstrated by examining RNA-seq data for the expression of genes associated with aggressive cancer behavior across cancer types (nCounter PanCancer Progression Genes) (Fig. 3) with the genes in the panel listed in Supplementary Table S2. The top 20 gene ontology (GO) terms associated with these genes are provided in Fig. 4.

The cancer grade did not differ between basal and luminal tumors. The median grade was 4 in tumors in both subtypes.

\section{Association between RNA-seq immune score and molecular subtype}

The immune scores consisted of: 11 cases with a score of 1 (non-T-cell-inflamed), 15 cases with a score of 2,11 cases with a score of 3,9 cases with a score of 4 , and 10 cases with a score of 5 (highest T-cellinflamed). The basal subtype was strongly associated with a T-cell-inflamed (immune infiltrated) signature in RNA-seq analyses (Fig. 5) with the genes in Fig. 5 listed in Supplementary Table S3. Of 27 basal tumors, 18 tumors $(66.7 \%)$ were immune-infiltrated with RNA-seq immune scores of 4-5, and the remaining nine tumors $(33.3 \%)$ had an immune score of 3. Of 29 luminal tumors, one tumor $(3.4 \%)$ had an immune score of 4 , two tumors $(6.9 \%)$ had an immune score of 3, and 26 tumors $(89.6 \%)$ were non-T-cell-inflamed with immune scores of 1-2. In multivariable analyses, dogs with a tumor immune score of 4-5 were much more like to have basal tumors (OR 52.22, 95\% CI 4.68-582.38, $P=0.001$ ). To further investigate changes in immune and related genes, heatmaps were generated using panels of specific genes involved in enhancing or suppressing 


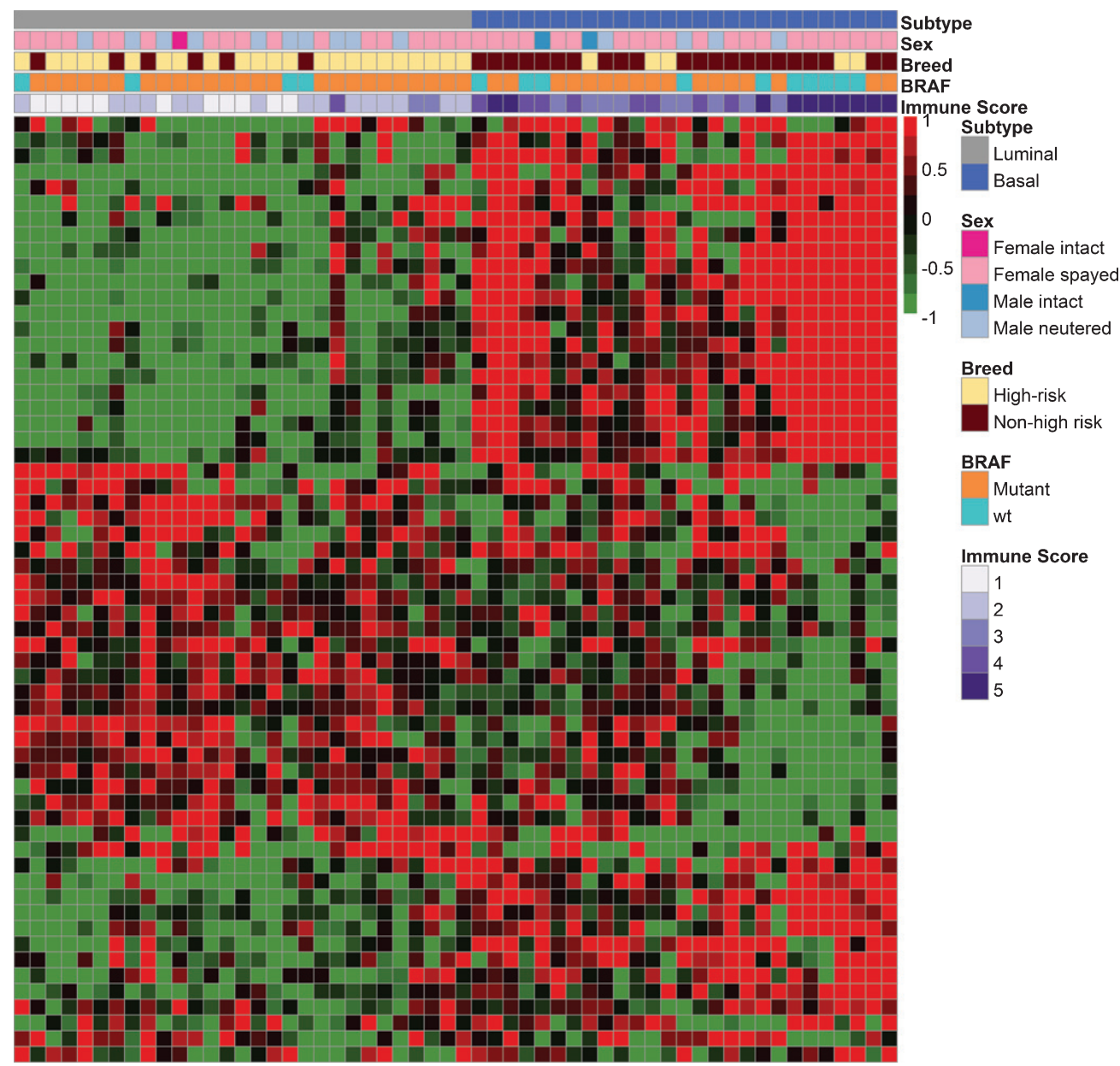

Fig. 1. Canine InvUC RNA-seq analyses demonstrating the presence of luminal ( $n=29$ cases) and basal ( $n=27$ cases) subtypes. A previously published 60-gene class prediction model was used for subtype assignment [21] of canine InvUC samples $(n=56)$ with comparison to canine normal bladder mucosa samples from dogs with no evidence of bladder disease $(n=4)$. In the heatmap, each column represents data from one sample, and each row represents a specific gene. The genes are listed in Supplementary Table S1 in the same order as they appear in the heatmap. Information regarding subtype, sex, breed, $B R A F$ status (mutated or not), and immune score is provided in the color bars above the heatmap. Note the segregation of tumor samples into two main groups identified as luminal and basal, with multiple subgroups within each subtype. Dogs in breeds with high genetic risk for InvUC were more likely to have luminal tumors, and dogs in other breeds to have basal tumors (OR 0.06 for basal tumors in high-risk breeds, $95 \% \mathrm{CI} 0.01-0.37, P=0.002)$. Basal tumors were strongly associated with immune infiltration (OR 52.22, $95 \%$ CI 4.68-582.38, $P=0.001)$ as indicated by purple bars, with darker shades indicating higher T-cell infiltration. There was an association between the $B R A F^{\mathrm{V} 595 \mathrm{E}}$ mutation and the luminal subtype, and between wild type $B R A F$ and the basal subtype (OR 0.27 for basal tumors having $B R A F^{\mathrm{V} 595 \mathrm{E}}, 95 \%$ CI $0.07-1.01, P=0.0424$ ).

the immune response to InvUC (Supplementary Figure S2), interferon-inducible genes (Supplementary Figure S3, Supplementary Table S4), and TGF- $\beta$ pathway related genes (Supplementary Figure S4, Supplementary Table S5). Overexpressed immunerelated genes included ones which typically enhance the immune response as well as others which keep the immune response in check (Supplementary Figure S2). $C D 8$ expression was prominent, especially in basal tumors. Thirty-nine tumors across subtypes expressed at least one checkpoint gene: $B 7 X, P D-L 1$,
$P D-1$ or $L A G-3$. Interferon-inducible gene signatures were mixed in the luminal and basal tumors, although some enrichment of IFN $\gamma$ genes in basal tumors was noted (Supplementary Figure S3). TGF- $\beta$ pathway signatures were enriched in basal tumors (Supplementary Figure S4).

Dogs in high-risk breeds were more likely to have low tumor immune scores (non-T-cell-inflamed), and dogs in non-high risk breeds were more likely to have higher tumor immune scores (T-cell-inflamed) (Mann Whitney U test, $P=0.0002$ ). Within high-risk 
A

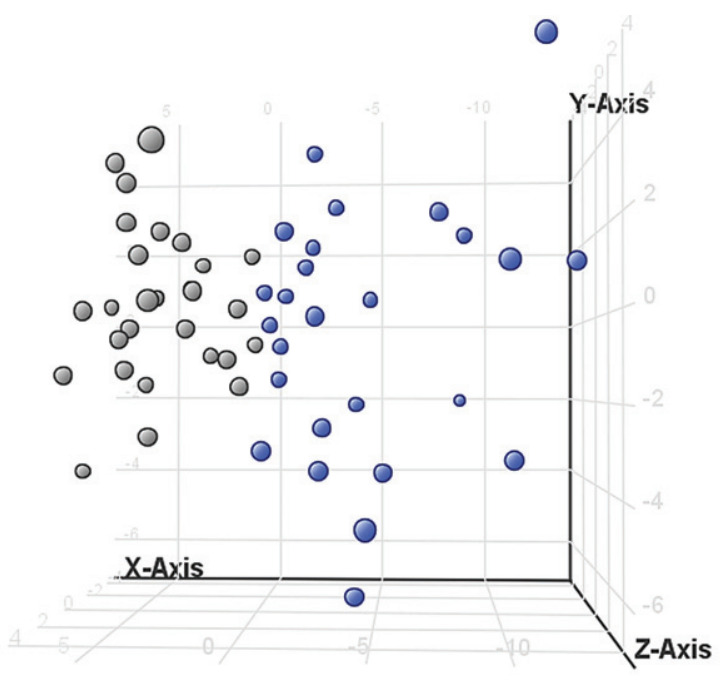

X-Axis

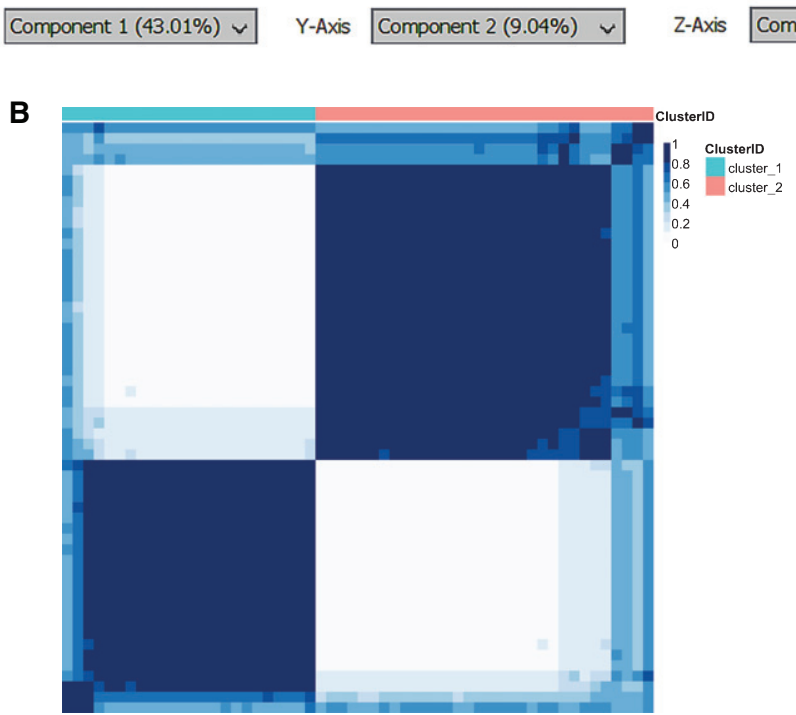

Fig. 2. Two subtypes identified as luminal and basal within canine InvUC samples. A. PCA plot using the 60-gene prediction model [21] demonstrates separation of the subtypes into two distinct groups. B. Consensus clustering demonstrates the stability of the two subtypes. Stability of subtypes assignment was verified by calculating their co-clustering frequency in iterative hierarchical clustering by applying consensus clustering ( $\mathrm{k}=2,1000$ bootstraps, 80\% subsamples), independent of the published class prediction model [30]. Results of the consensus clustering are displayed in the figure. The consensus score is denoted with values ranging from 0 (never clustered together) to 1 (always clustered together) and marked by white to dark blue colors. Two distinct clusters containing $90 \%$ of the samples with high ( $>0.8$ ) consensus score were observed. Approximately $\sim 10 \%$ of the samples were unstable with low $(<0.8)$ consensus score. Consensus clustering was also performed with up to $\mathrm{K}=10$, forcing the creation of maximum 10 groups. Approximately $60 \%$ of the samples were retained in the two distinct groups indicating high stability.

breed dogs, the immune scores were: $1(n=9 \mathrm{dogs})$, $2(n=11 \mathrm{dogs}), 3(n=4 \mathrm{dogs}), 4(n=2 \mathrm{dogs})$, and 5 ( $n=2$ dogs). Within non-high risk breed dogs, the immune scores were: $1(n=2 \mathrm{dogs}), 2(n=4 \mathrm{dogs})$, $3(n=7 \mathrm{dogs}), 4(n=7 \mathrm{dogs})$, and $5(n=8 \mathrm{dogs})$. The association between RNA-seq immune score and subtype was present across breeds. Amongst the 28 high-risk breed dogs, T-cell-inflamed tumors (immune score 4-5) were much more likely to be basal than luminal (OR 33.00, 95\% CI 2.25-484.45, $P=0.0047$ ). Within the 28 non-high risk breed dogs, all 15 T-cell-inflamed tumors were also in the basal subtype. The 13 non-T-cell-inflamed tumors in the non-high risk breed dogs included six luminal tumors 
Table 2

Association between subtype and clinical and pathological variables listed. The data are reported in relation to the basal subtype

\begin{tabular}{|c|c|c|c|c|}
\hline Variable & Level & Odds Ratio & $95 \% \mathrm{CI}$ & $P$-value \\
\hline \multicolumn{5}{|l|}{ Univariable analyses } \\
\hline High Risk Breed & Yes & 0.06 & $0.02-0.22$ & $<0.0001$ \\
\hline Sex & Male & 0.54 & $0.17-1.78$ & 0.308 \\
\hline Age & Years & 1.22 & $0.94-1.59$ & 0.114 \\
\hline Bladder cancer screening & Yes & 0.38 & $0.07-2.17$ & 0.2584 \\
\hline$B R A F$ & $B R A F^{V 595 E}$ mutant & 0.27 & $0.07-1.01$ & 0.0424 \\
\hline RNA-seq immune score & $\begin{array}{l}\text { Score } 4-5 \text { (T-cell- inflamed) } \\
\text { compared to scores of } 1 \text { to } 3\end{array}$ & 56.00 & $6.53-480.35$ & $<0.0001$ \\
\hline Tumor grade & $\begin{array}{l}\text { Higher grade compared to } \\
\text { lower grade }\end{array}$ & 0.99 & $0.39-2.52$ & 0.9873 \\
\hline $\mathrm{T}$ stage at diagnosis & $\mathrm{T} 3$ & 1.79 & $0.44-7.18$ & 0.4140 \\
\hline $\mathrm{N}$ stage at diagnosis & $\mathrm{N} 1$ & 1.08 & $0.20-5.89$ & 0.9262 \\
\hline M stage at diagnosis & M1 & 3.50 & $0.34-35.90$ & 0.2573 \\
\hline M stage at death & M1 & 2.53 & $0.71-8.97$ & 0.1465 \\
\hline Apex & Yes & 1.12 & $0.37-3.34$ & 0.8420 \\
\hline Mid bladder & Yes & 1.56 & $0.54-4.49$ & 0.4096 \\
\hline Trigone & Yes & 1.68 & $0.55-5.08$ & 0.3579 \\
\hline Urethra & Yes & 1.36 & $0.47-3.91$ & 0.5706 \\
\hline Prostate & Yes & 0.50 & $0.06-4.47$ & 0.5308 \\
\hline More than 1 location & Yes & 3.60 & $0.86-15.12$ & 0.0639 \\
\hline Tumor volume & Larger volume (cm3) & 1.01 & $0.96-1.06$ & 0.6939 \\
\hline \multicolumn{5}{|l|}{ Multivariable analyses } \\
\hline High-Risk Breed & Yes & 0.06 & $0.01-0.37$ & 0.002 \\
\hline RNA-seq immune score & $\begin{array}{l}\text { Score } 4-5 \text { compared } \\
\text { to score of } 1-3\end{array}$ & 52.22 & $4.68-582.38$ & 0.001 \\
\hline
\end{tabular}

and seven basal tumors, although a significant association between immune score and subtype within non-high risk breed dogs was not found. An association between the CD3 IHC findings and the RNA-seq immune patterns was not detected, and the CD3 IHC immune score did not correlate with subtype.

Seven Scottish Terriers had bladder cancer identified through screening, and of those seven dogs, three $(42.7 \%)$ had T-cell-inflamed tumors with immune scores of 4-5, two (28.6\%) had an immune score of 3 , and two $(28.6 \%)$ had non-T-cell-inflamed tumors with immune scores of 1-2. Twelve Scottish Terriers had not undergone screening and were presented with urinary signs. This included two dogs $(16.7 \%)$ with T-cell-inflamed tumors with immune scores of 4-5, two dogs (16.7\%) with an immune score of 3 , and eight dogs $(66.7 \%)$ with immune scores of 1-2. With the small numbers of dogs, the potential association between higher immune scores in dogs screened for InvUC was not significant (OR 2.4, 95\% CI $0.42-$ 3.57, $P=0.3026$ ).

\section{Association between BRAF $F^{V 55 E}$ mutation and molecular subtype}

The $B R A F^{V 595 E}$ mutation was present in the tumor in $42(75 \%)$ of the 56 dogs. There was an association between the $B R A F^{V 595 E}$ mutation and the luminal subtype, and between wild type $B R A F$ and the basal subtype in univariable analyses (OR 0.27 for basal tumors having $B R A F^{V 595 E}, 95 \%$ CI $0.07-1.01$, $P=0.0424)$. Of 14 tumors with wild type $B R A F, 10$ tumors $(71.4 \%)$ were basal, and four tumors $(28.6 \%)$ were luminal. Of 42 tumors with the $B R A F^{V 595 E}$ mutation, 17 tumors $(40.5 \%)$ were basal, and 25 tumors $(59.5 \%)$ were luminal.

Tumors with the $B R A F^{V 595 E}$ mutation were often present in dogs in high-risk breeds. A total of $28 \mathrm{dogs}$ were in high-risk breeds, 23 of which (82\%) had the $B R A F^{V 595 E}$ mutation. Of 24 terriers, $21(87.5 \%)$ had the $B R A F^{V 595 E}$ mutation. The $B R A F^{V 595 E}$ mutation was also present in $67.9 \%$ of dogs not in high-risk breeds. There was evidence, however, that the breedrelated effect on subtype as discussed above persisted in wild type and mutant tumors. Of 19 dogs in lowrisk breeds, i.e. breeds associated with basal tumors above, 14 had basal tumors even in the presence of $B R A F$ mutations. Similarly, of five dogs in high risk breeds, i.e. breeds associated with luminal tumors above, three of five dogs had luminal tumors even in the presence of wild type BRAF. In a subset analysis, the tumors in seven Scottish Terriers screened for bladder cancer did not differ from the tumors in 12 Scottish Terriers not screened for bladder cancer in regards to subtype or $B R A F$ mutation status. 


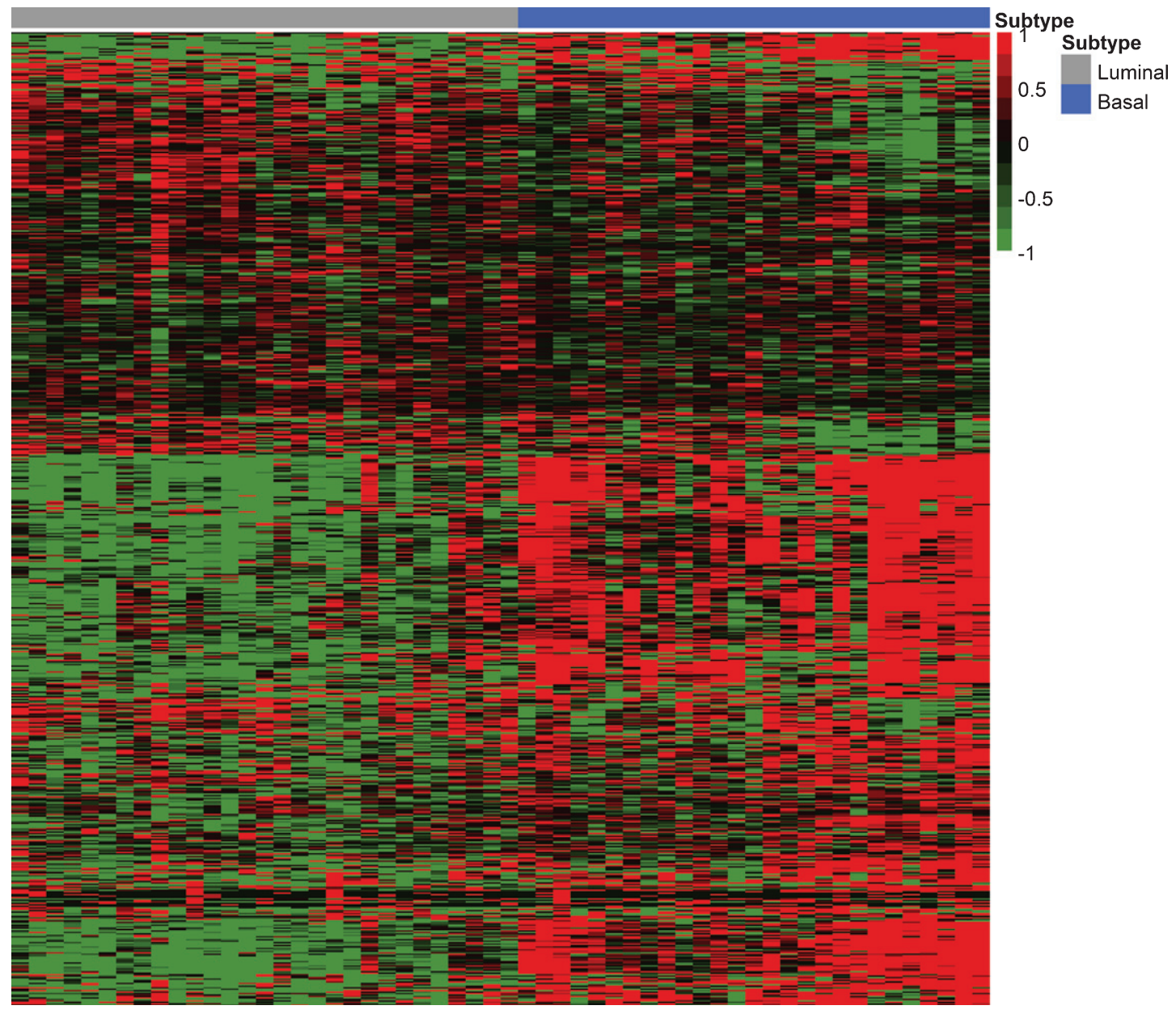

Fig. 3. Canine InvUC RNA-seq analyses. In the heatmap, the luminal and basal subtype tumors (gray and blue column headings, respectively) were defined by class prediction model [21]. The data were interrogated for genes associated with cancer progression across cancer types (nCounter ${ }^{\circledR}$ PanCancer Progression Gene Panel, $n=770$ genes, Nanostring Technologies, Seattle, WA). Genes enriched in canine InvUC tumors were queried against these genes (canine orthologs identified for $n=669$ genes) which are listed in Supplementary Table S2 in the same order as in the heatmap. Note the strong enrichment for genes associated with cancer progression in the canine InvUC samples. The top most significant gene ontology (GO) terms associated with these genes $(n=20)$ are summarized in Fig. 4.

When interrogating the InvUC RNA-seq data with specific panels of genes reported to be enriched in tumors with the $B R A F^{V 595 E}$ mutation, or in wild type $B R A F$ tumors [33], the gene expression patterns followed the published results (Supplementary Figure S5) with genes listed in Supplementary Table S6. These gene expression patterns around mutation status were not affected by breed-risk or subtype.

\section{DISCUSSION}

This study demonstrated several similarities between dogs and humans regarding clinical and tumor characteristics related to InvUC subtypes.
One of the most critical findings was the association between the molecular subtype and immune signatures. T-cell-inflamed signatures were strongly associated with the basal subtype, and non-T-cellinflamed signatures were associated with the luminal subtype. These findings in dogs parallel findings in InvUC in humans in which tumors in the basal subtype have increased infiltration of CD8 + T cells and T-cell-inflamed signatures in RNA-seq analysis [9, 11, 15-17]. The association between a T-cellinflamed state and the basal subtype was present in dogs from high-risk breeds for InvUC, as well as in dogs in other breeds. This result is especially important in light of the fact that the immune state 


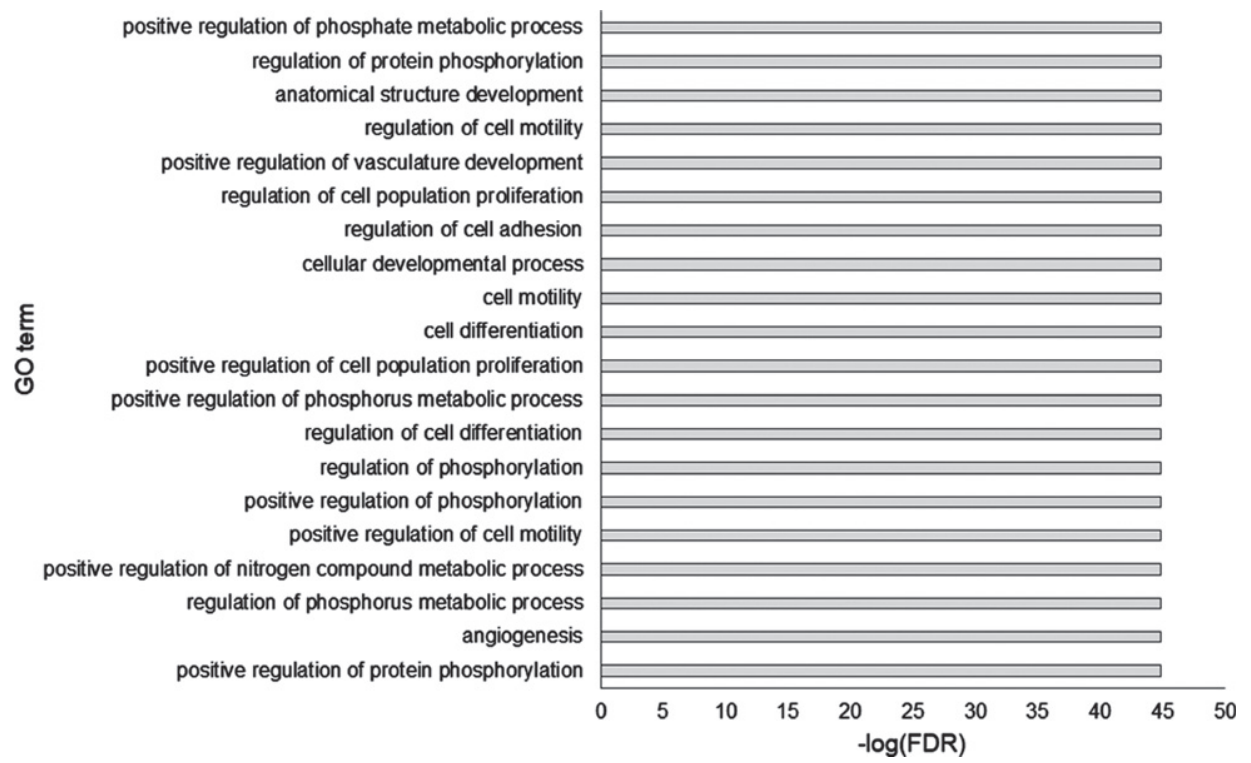

Fig. 4. Gene ontology (GO) analyses of the 669 cancer progression genes in Fig. 3. GO analyses were conducted in Strand NGS applying Fisher exact test with multiple test correction (Bonferroni) [35]. The top $20 \mathrm{GO}$ terms ( $\mathrm{p}$ corr $<0.001$ ) associated with biological processes were compiled. The $\mathrm{x}$-axis indicates $-\log (\mathrm{FDR})$ values associated with the GO terms indicated on the $\mathrm{y}$-axis.

of the tumor can strongly affect whether the patient responds or fails to respond to multiple different types of cancer therapies $[7,9,15,31,26]$. Immune infiltrated tumors are more sensitive to immunotherapies, as well as chemotherapy and radiation therapy, whereas non-infiltrated tumors are typically less responsive $[9,15,41]$. This indicates that the canine InvUC model could be informative in multiple ways. Dogs could be included in studies to optimize therapies in the context of T-cell-inflamed tumors. Dogs with non-T-cell-inflamed tumors could be included in other studies to test strategies to promote the infiltration of immune cells into the tumor, i.e. to convert immune cold tumors to immune hot tumors in order to improve treatment responses.

As anticipated, the immune infiltrative signatures included the expression of genes which would enhance the immune response against the cancer as well as genes which would dampen the immune response. Both are typically present in T-cell-inflamed tumors in humans [31, 41, 42]. A mixture of pro-immune processes along with the expression of immune checkpoints and TGF- $\beta$ pathway genes was noted in the canine samples. This suggests that the canine tumors could be well-suited for the study of checkpoint inhibitors, other immunotherapies, and combination therapies in an environment similar to that in human InvUC.
The expression of TGF- $\beta$ in the canine tumors was considered an important finding in relation to recent attention to the effects of stromal cell expression of TGF- $\beta$ in preventing the infiltration of immune cells into the tumor and limiting the activity of immune checkpoint inhibitors [11,43]. Future singlecell sequencing studies could provide an expanded understanding of this pathway and others in the dog tissues. In addition to the effects of stromal TGF- $\beta$, for example, TGF- $\beta$ expressed in the tumor cells can inhibit the growth of early bladder cancer and drive the progression of later cancer [11, 43-45].

An interesting finding, albeit with small numbers of dogs for study, was the immune signature in dogs in which the cancer was identified through screening rather than after clinical signs emerged. Of seven Scottish Terriers with cancer detected through screening, five dogs $(71.4 \%)$ had immune scores of 3-5, and two dogs $(28.6 \%)$ had immune scores of $1-2$. Of 12 Scottish Terriers who were presented with typical "symptomatic" cancer, only four dogs (33.3\%) had immune scores of 3-5, and eight dogs $(66.7 \%)$ had immune scores of 1-2. This finding, if confirmed in larger numbers of dogs, could be in line with the expectation that antitumor immune processes in early tumors are more active than in later stage cancers [46].

The numbers of tumor infiltrating lymphocytes identified by CD3 IHC did not correlate with the 


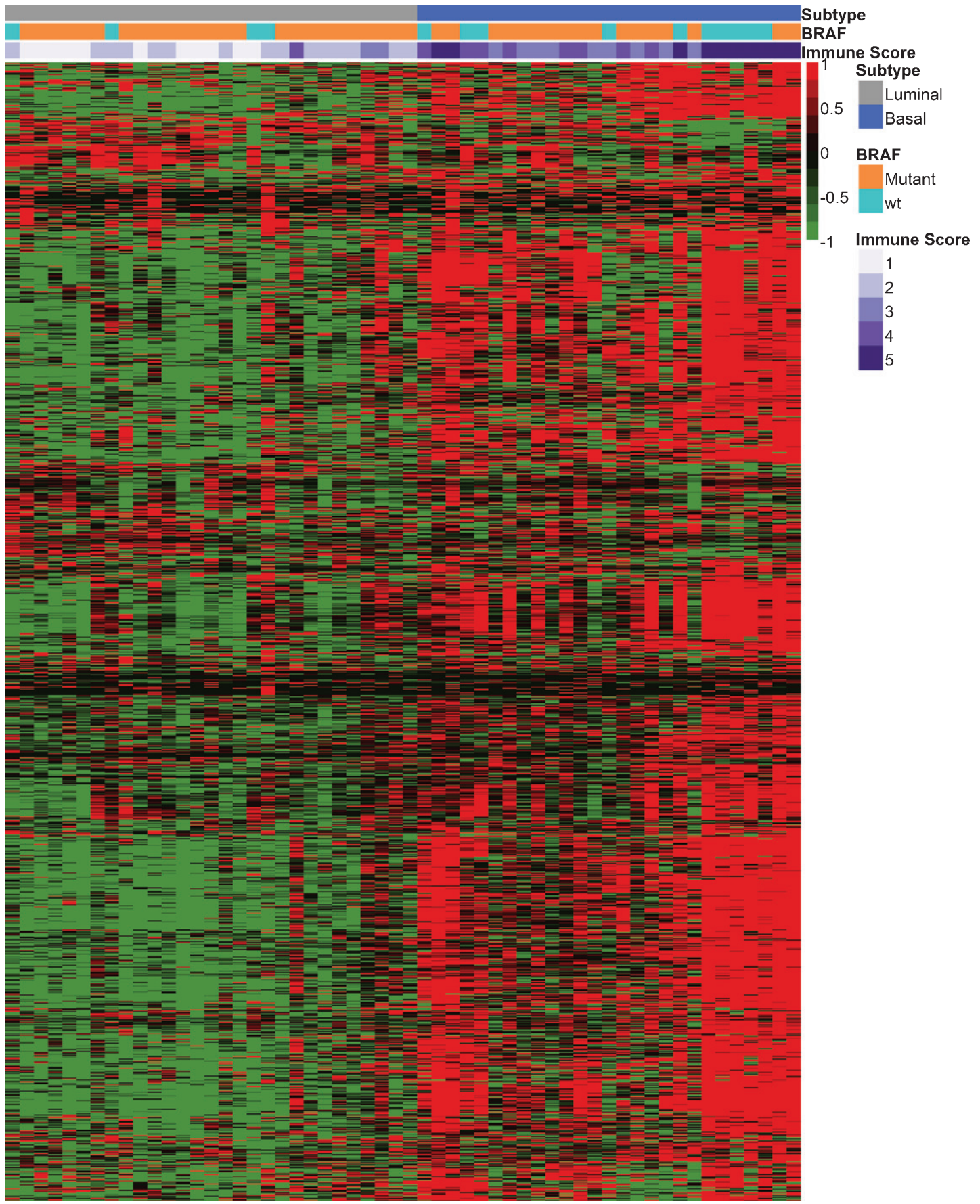

Fig. 5. Canine InvUC RNA-seq analyses. In the heatmap, the luminal and basal subtype samples (gray and blue column headings, respectively) were defined by class prediction model [21]. The data were interrogated for genes that classify human InvUC as T-cell-inflamed $(n=1797)$ [31]. Note that the expression of genes related to immune infiltration (red color in the heatmap) predominate in the basal subtype tumors (OR $52.22,95 \%$ CI 4.68-582.38, $P=0.001$ ). The genes in the heatmap are listed in Supplementary Table S3 in the same order as they appear in the figure.

RNA-seq immune score, although this was not surprising. IHC findings would be based on the tissue slice where the tumor was cut for slide preparation, whereas the RNA-seq score would be representative of multiple layers through the tissue. It is possible that the IHC findings could be more representative if sev- 
eral slides from each tumor were examined, but this was not usually possible due to the small size of cystoscopic biopsies which can be obtained from dogs. In this study with limited size biopsies, the RNA-seq analyses were prioritized over IHC as the RNA-seq analyses were expected to provide considerably more information. Another current limitation in using IHC to characterize the immune cells in canine tissues is the lack of important antibodies for formalin-fixed specimens, such as the lack of specific antibodies to canine CD8.

Another key finding from the study was the very strong association between subtype and dog breed, specifically the association between dogs in highrisk breeds for InvUC and luminal subtype cancer. This strongly suggests that inherited genetic variation influences the subtype of InvUC that develops. This is not entirely unexpected. In human breast cancer, in which luminal and basal molecular subtypes were first described, germline variants along with patient exposures, such as physiological changes associated with breast feeding, have been linked to the development of specific subtypes of the cancer [47]. In other work, the ancestry of patients and ancestryrelated genes have been linked to multiple types of human cancer including bladder cancer, and these analyses are especially informative when focused at the level of molecular subtypes [48]. While germline mutations have been incriminated in $\sim 14 \%$ of cases of InvUC in humans, a possible link between these specific germline mutations and the emergence of specific cancer subtypes has not been reported [49, 50]. Work has been focused more on mutation signatures related to exposure to cigarette smoke and chemicals [51].

There is strong interest in better defining inherited, as well as acquired genetic variants that increase InvUC risk in order to develop new strategies to prevent InvUC and to manage it more effectively when it develops. The diversity of germline genes in humans creates challenges in defining specific inherited variants that are most important in a given disease process. Dogs in specific breeds, however, offer a unique opportunity to study genetic variations linked to cancer [52-54]. Most dog breeds have been established in the last 300 years, with many breeds derived from a small number of founder dogs, and most having undergone strong artificial selection for specific traits such as size, shape, fur type, etc. [52, 53]. Deleterious alleles can "hitch-hike" along with those linked to desired traits defined by dog breeders. The registration process for dogs in specific breeds has contributed heavily to a relatively closed gene pool within each breed. This has inadvertently led to a high level of breed-associated genetic disease including cancer [52-54]. With the compressed lifespan of dogs, tracking environmental exposures can also be accomplished more effectively than in humans $[23,54]$. Thus, dogs offer important models to discover the genomics driving cancer susceptibility, subtypes, and behavior, as well as models for targeted therapies and immunotherapies [54].

Several clinical features that have been associated with a specific subtype of human InvUC were assessed in the dogs in the study. Women, for example, are more likely than men to have basal subtype InvUC [7-9]. Female dogs were overrepresented in dogs with basal tumors with $78 \%$ percent of dogs being female, although $65 \%$ of dogs with luminal tumors were also female. Of 40 female dogs, however, 39 dogs had been spayed, and this could have impacted potential effects of sex on subtype, as well as the consistent predilection for female dogs in the development of InvUC in general [22, 23].

In humans, the basal subtype of InvUC is associated with more advanced stage at diagnosis, and more frequent development of distant metastases [7-9]. Although distant metastases at diagnosis were uncommon in dogs in this study, it was noted that three of four dogs with distant metastases at diagnosis had basal tumors. The presence of distant metastases at death was considered an exploratory endpoint because treatments differed between dogs, yet it was noted that $53 \%$ of dogs with basal tumors had distant metastases, compared to $32 \%$ dogs with luminal tumors. The presence of distant metastases early in the disease course is another indicator of the aggressiveness of the cancer. In exploratory analyses, the median time between diagnosis and the detection of distant metastasis was approximately three times longer in dogs with luminal tumors compared to dogs with basal tumors $(P=0.0113)$. Clearly, studies of larger numbers of dogs and groups of dogs treated with a specific treatment protocol are indicated to further assess the relationship between subtype, cancer stage, and case outcomes.

The $B R A F^{V 595 E}$ mutation in InvUC was detected in $75 \%$ of the dogs in this study, which is consistent with earlier studies $[24,25]$. The $B R A F^{V 595 E}$ mutation was significantly associated with the luminal subtype, and wild type $B R A F$ with the basal 
subtype, although there was clearly overlap between the groups. The mutation, for example was present in $86 \%$ of dogs with luminal tumors, but was also present in $63 \%$ of dogs with basal tumors. Similarly, of 14 dogs with wild type tumors, $10 \operatorname{dogs}(71 \%)$ had basal tumors, and four dogs (29\%) had luminal tumors. Genes that are oppositely regulated between $B R A F^{V 595 E}$ mutant and BRAF wild type InvUC samples in dogs have been described, and the patterns of expression of these genes around $B R A F$ status was confirmed in the current study [33].

This study focused on the major subtypes of luminal and basal tumors. In human InvUC subgroupings within the luminal and basal subtypes are consistently reported, such as luminal papillary/uroA, luminal infiltrated/p53-like, luminal/genomically unstable, basal squamous, and neuroendocrine [7-11]. It is likely that similar subgroupings could occur in canine InvUC, and in fact there was evidence for the presence of subgrouping within the tumors in this study. Analyses with larger numbers of cases will help further elucidate the subgrouping in canine tumors.

In addition to the results of the analyses reported here, the data from this study, as well as additional canine InvUC data from other work have been deposited in the National Cancer Institute's Integrated Canine Data Commons (https://datacom mons.cancer.gov/ repository/integrated-canine-datacommons), a cloud based resource in which many different types of data including genomic, clinical, outcome, and pharmacokinetic data are accessible. These canine data sets are available along with data from the human Cancer Data Commons, offering the opportunity for robust cross species comparisons in the cloud based system. Building on the existing data and data currently being generated, several key questions could be addressed in the future related to the effects of subtype on specific therapies, the effects of specific therapies in modulating subtype, i.e. switching from one subtype to another, and the effects of germline variants in the emergence of specific subtypes. The immunological findings will be of additional importance when immunotherapies such as immune checkpoint inhibitors, and others are established and are made available for canine studies. In conclusion, this study demonstrated clinical and tumor characteristics that were associated with molecular subtypes across canine and human samples, and provides further support for the value for the naturally-occurring canine InvUC model for translational research.

\section{ACKNOWLEDGMENTS}

The authors wish to thank the dedicated clinicians and staff of the Purdue Comparative Oncology Program for their work on this study, and the dog owners who allowed their dogs to participate and provided support for bladder cancer research at Purdue University.

\section{FUNDING}

National Institutes of Health, P30 Administrative Supplement Award: "Advancing immunology in dogs with naturally-occurring invasive bladder cancer, a relevant model to improve immunotherapy across molecular cancer subtypes in humans" to Parent CCSG Project: P30CA023168, Purdue University Center for Cancer Research, Investigators TL Ratliff and DW Knapp.

\section{AUTHOR CONTRIBUTIONS}

Breann C. Sommer: conception, performance of work, interpretation and analysis of data, writing the article. Deepika Dhawan: conception; performance of work, interpretation and analysis of data, writing the article. Audrey Ruple: performance of work, interpretation and analysis of data, writing the article. José A. Ramos-Vara: performance of work, interpretation and analysis of data, writing the article. Noah M. Hahn: conception, interpretation and analysis of data, writing the article. Sagar M. Utturkar: performance of work, interpretation and analysis of data, writing the article. Elaine A. Ostrander: interpretation and analysis of data, writing the article. Heidi G. Parker: interpretation and analysis of data, writing the article. Christopher M. Fulkerson: performance of work, interpretation of data, writing the article. Michael O. Childress: performance of work, interpretation of data, writing the article. Lindsey M. Fourez: performance of work, writing the article. Alexander W. Enstrom: performance of work, writing the article. Deborah W. Knapp: conception, performance of work, interpretation and analysis of data, writing the article. All authors had access to the data.

\section{CONFLICT OF INTEREST}

Breann C. Sommer, Deepika Dhawan, Audrey Ruple, José A. Ramos-Vara, Sagar M. Utturkar, 
Elaine A Ostrander, Heidi G. Parker, Christopher M. Fulkerson, Michael O. Childress, Lindsey M. Fourez, Alexander W. Enstrom, and Deborah W. Knapp have nothing to disclose. Dr. Hahn reports personal fees and non-financial support from Genentech, personal fees and non-financial support from Merck, non-financial support from BMS, personal fees and non-financial support from Seattle Genetics, personal fees and non-financial support from Incyte, non-financial support from Inovio, personal fees and non-financial support from Janssen, personal fees and non-financial support from Pieris, personal fees from Boehringer Ingelheim, personal fees from Glaxo Smith Kline, personal fees from Bladder Cancer Academy, personal fees from Ferring, nonfinancial support from AstraZeneca, non-financial support from HTG Molecular, personal fees from Pfizer, outside the submitted work.

\section{SUPPLEMENTARY MATERIAL}

The supplementary material is available in the electronic version of this article: https://dx.doi.org/ 10.3233/BLC-201523.

\section{REFERENCES}

[1] Siegel RL, Miller KD, Jemal A. Cancer statistics, 2020. CA Cancer J Clin. 2020;70(1):7-30.

[2] Czerniak B, Dinney C, McConkey D. Origins of bladder cancer. Annu Rev Pathol. 2016;11:149-74.

[3] Hahn NM. New treatments for bladder cancer. Clin Adv Hematol Oncol. 2016;14(11):884-87.

[4] Patel VG, Oh WK, Galsky MD. Treatment of muscleinvasive and advanced bladder cancer in 2020. CA Cancer J Clin. 2020 Aug 7 [cited 2020 Nov 11]. doi: 10.3322/caac.21631. Online ahead of print.

[5] Alhalabi O, Campbell M, Shah A, Siefker-Radtke A, Gao J. Emerging treatments in advanced urothelial cancer. Curr Opin Oncol. 2020;32(3):232-39.

[6] Thana M, Wood L. Immune checkpoint inhibitors in genitourinary malignancies. Curr Oncol. 2020;27(Suppl 1):S69-S77.

[7] Robertson AG, Kim J, Al-Ahmadie H, Bellmunt J, Guo G, Cherniack AD, et al. Comprehensive molecular characterization of muscle-invasive bladder cancer. Cell. 2017;171(3):540-556.e25.

[8] Choi W, Porten S, Kim S, Willis D, Pilmack ER, HoffmanCensits J, et al. Identification of distinct basal and luminal subtypes of muscle-invasive bladder cancer with different sensitivities to frontline chemotherapy. Cancer Cell. 2014;25(2):152-65.

[9] McConkey DJ, Choi W. Molecular subtypes of bladder cancer. Curr Oncol Rep. 2018;20(10):77.

[10] McConkey DJ, Choi W, Shen Y, Lee IL, Porten S, Matin SF, et al. A prognostic gene expression signature in the molecular classification of chemotherapy-naive urothelial cancer is predictive of clinical outcomes from neoadjuvant chemotherapy: a phase 2 trial of dose-dense methotrexate, vinblastine, doxorubicin, and cisplatin with bevacizumab in urothelial cancer. Eur Urol. 2016;69(5): 855-62.

[11] Sjödahl G, Jackson CL, Bartlett JM, Siemens DR, Berman DM. Molecular profiling in muscle-invasive bladder cancer: more than the sum of its parts. J Pathol. 2019;247(5): 563-73.

[12] Fong MHY, Feng M, McConkey DJ, Choi W. Update on bladder cancer molecular subtypes. Transl Androl Urol. 2020;9(6):2881-9.

[13] Bernardo C, Eriksson P, Marzouka NA, Liedberg F, Sjödahl G, Höglund M. Molecular pathology of the luminal class of urothelial tumors. J Pathol. 2019;249(3):308-18.

[14] Necchi A, Raggi D, Gallina A, Ross JS, Farè E, Giannatempo $\mathrm{P}$, et al. Impact of molecular subtyping and immune infiltration on pathological response and outcome following neoadjuvant pembrolizumab in muscle-invasive bladder cancer. Eur Urol. 2020;77(6):701-10.

[15] Seiler R, Ashab HAD, Erho N, van Rhijn BWG, Winters B, Douglas J, et al. Impact of molecular subtypes in muscleinvasive bladder cancer on predictive response and survival after neoadjuvant chemotherapy. Eur Urol. 2017;72(4):54454.

[16] Hodgson A, Liu SK, Vesprini D, Xu B, Downes MR. Basalsubtype bladder tumours show a 'hot' immunophenotype. Histopathology. 2018;73(5):748-57.

[17] Ren R, Tyryshkin K, Graham CH, Koti M, Siemens DR. Comprehensive immune transcriptomic analysis in bladder cancer reveals subtype specific immune gene expression patterns of prognostic relevance. Oncotarget. 2017;8(41):70982-71001.

[18] Mak IW, Evaniew N, Ghert M. Lost in translation: animal models and clinical trials in cancer treatment. Am J Transl Res. 2014;6(2):114-8.

[19] Le Magnen C, Dutta A, Abate-Shen C. Optimizing mouse models for precision cancer prevention. Nat Rev Cancer. 2016;16(3):187-96.

[20] Sommer BC, Dhawan D, Ratliff TL, Knapp DW. Naturally-occurring canine invasive urothelial carcinoma: a model for emerging therapies. Bladder Cancer. 2018;4(2): 149-59.

[21] Dhawan D, Hahn NM, Ramos-Vara JA, Knapp DW. Naturally-occurring canine invasive urothelial carcinoma harbors luminal and basal transcriptional subtypes found in human muscle invasive bladder cancer. PLoS Genet. 2018;14(8):e1007571.

[22] Knapp DW, Dhawan D, Ramos-Vara JA, Ratliff TL, Cresswell GM, Utturkar S, et al. Naturally-occurring invasive urothelial carcinoma in dogs, a unique model to drive advances in managing muscle invasive bladder cancer in humans. Front Oncol. 2020;9:1493.

[23] Knapp DW, Ramos-Vara JA, Moore GE, Dhawan D, Bonney PL, Young KE. Urinary bladder cancer in dogs, a naturally occurring model for cancer biology and drug development. ILAR J. 2014;55(1):100-18.

[24] Decker B, Parker HG, Dhawan D, Kwon EM, Karlins E, Davis BW, et al. Homologous mutation to human BRAF V600E is common in naturally occurring canine bladder cancer - evidence for a relevant model system and urine-based diagnostic test. Mol Cancer Res. 2015;13(6):993-1002.

[25] Mochizuki H, Shapiro SG, Breen M. Detection of BRAF mutation in urine DNA as a molecular diagnostic for 
canine urothelial and prostatic carcinoma. PLoS One. 2015;10(12):e0144170.

[26] Longo T, McGinley KF, Freedman JA, Etienne W, Wu Y, Sibley A, Owzar K, Gresham J, Moy C, Szabo S, Greshock J, Zhou H, Bai Y, Inman BA. Targeted Exome Sequencing of the Cancer Genome in Patients with Very High-risk Bladder Cancer. Eur Urol. 2016;70(5):714-17.

[27] Nahm KY, Heo JS, Lee JH, Lee DY, Chung KR, Ahn $\mathrm{HW}$, et al. Gene profiling of bone around orthodontic miniimplants by RNA-sequencing analysis. Biomed Res Int. 2015;2015:538080.

[28] Love MI, Huber W, Anders S. Moderated estimation of fold change and dispersion for RNA-seq data with DESeq2. Genome Biology. 2014;15(12):550.

[29] Robinson MD, Oshlack A. A scaling normalization method for differential expression analysis of RNA-seq data. Genome Biology. 2010;11(3):R25.

[30] Wilkerson MD, Hayes DN. ConsensusClusterPlus: a class discovery tool with confidence assessments and item tracking. Bioinformatics. 2010;26(12):1572-3.

[31] Sweis RF, Spranger S, Bao R, Paner GP, Stadler WM, Steinberg G, et al. Molecular drivers of the non-T-cell-inflamed tumor microenvironment in urothelial bladder cancer. Cancer Immunol Res. 2016;4(7):563-8.

[32] Kanehisa M, Goto S. KEGG: kyoto encyclopedia of genes and genomes. Nucleic Acids Res. 2000;28(1):27-30.

[33] Parker HG, Dhawan D, Harris AC, Ramos-Vara JA, Davis BW, Knapp DW, Ostrander EA. RNAseq expression patterns of canine invasive urothelial carcinoma reveal two distinct tumor clusters and shared regions of dysregulation with human bladder tumors. BMC Cancer. 2020;20(1): 251.

[34] Dong L, Huang CY, Johnson EJ, Yang L, Zieren RC, Horie $\mathrm{K}$, et al. High-throughput simultaneous mRNA profiling using nCounter technology demonstrates that extracellular vesicles contain different mRNA transcripts than their parental prostate cancer cells. Anal Chem. 2021;93(8):3717-25.

[35] Ashburner M, Ball CA, Blake JA, Botstein D, Butler H, Cherry JM, et al. Gene ontology: tool for the unification of biology. Nat Genet. 2000;25(1):25-9.

[36] Owen LN, Unit WHOVPH, O. TNM Classification of Tumours in Domestic Animals/ edited by L.N. Owen. World Health Organization; 1980 [cited 2020 Aug 13]; Available from: https://apps.who.int/iris/handle/10665/68618

[37] Knapp DW, Glickman NW, Denicola DB, Bonney PL, Lin TL, Glickman LT. Naturally-occurring canine transitional cell carcinoma of the urinary bladder A relevant model of human invasive bladder cancer. Urol Oncol. 2000;5(2):4759.

[38] Cheng L, MacLennan GT, Lopez-Beltran A. Histologic grading of urothelial carcinoma: a reappraisal. Hum Pathol. 2012;43(12):2097-108.

[39] Webster JD, Miller MA, DuSold D, Ramos-Vara JA. Effects of prolonged formalin fixation on diagnostic immunohistochemistry in domestic animals. J Histochem Cytochem. 2009;57(8):753-61.
[40] Sharma P, Shen Y, Wen S, Yamada S, Jungbluth AA, Gnjatic $\mathrm{S}$, et al. CD8 tumor-infiltrating lymphocytes are predictive of survival in muscle-invasive urothelial carcinoma. Proc Natl Acad Sci USA. 2007;104(10):3967-72.

[41] Galluzzi L, Zitvogel L, Kroemer G. Immunological mechanisms underneath the efficacy of cancer therapy. Cancer Immunol Res. 2016;4(11):895-902.

[42] Joseph M, Enting D. Immune responses in bladder cancerrole of immune cell populations, prognostic factors and therapeutic implications. Front Oncol. 2019;9:1270.

[43] Sanjabi S, Oh SA, Li MO. Regulation of the Immune Response by TGF- $\beta$ : From conception to autoimmunity and infection. Cold Spring Harb Perspect Biol. 2017;9(6):a022236.

[44] Ciardiello D, Elez E, Tabernero J, Seoane J. Clinical development of therapies targeting TGF $\beta$ : current knowledge and future perspectives. Ann Oncol. 2020;31(10):1336-49.

[45] Zou J, Huang R, Li H, Wang B, Chen Y, Chen S, Ou K, Wang $\mathrm{X}$. Secreted TGF-beta-induced protein promotes aggressive progression in bladder cancer cells. Cancer Manag Res. 2019;11:6995-7006.

[46] Chen DS, Mellman I. Elements of cancer immunity and the cancer-immune set point. Nature. 2017;541(7637):321-30.

[47] Holm J, Eriksson L, Ploner A, Eriksson M, Rantalainen M, Li J, Hall P, Czene K. Assessment of Breast Cancer Risk Factors Reveals Subtype Heterogeneity. Cancer Res. 20171;77(13):3708-17.

[48] Carrot-Zhang J, Chambwe N, Damrauer JS, Knijnenburg TA, Robertson AG, Yau C, et al. Cancer Genome Atlas Analysis Network, Cherniack AD, Beroukhim R. Comprehensive analysis of genetic ancestry and its molecular correlates in cancer. Cancer Cell. 2020;37(5):639-654.e6.

[49] Vlachostergios PJ, Faltas BM, Carlo MI, Nassar AH, Alaiwi SA, Sonpavde G. The emerging landscape of germline variants in urothelial carcinoma: Implications for genetic testing. Cancer Treat Res Commun. 2020;23:100165.

[50] Carlo MI, Ravichandran V, Srinavasan P, Bandlamudi C, Kemel Y, Ceyhan-Birsoy O, et al. Cancer susceptibility mutations in patients with urothelial malignancies. J Clin Oncol. 2020;38(5):406-14.

[51] Hayashi T, Fujita K, Hayashi Y, Hatano K, Kawashima A, McConkey DJ, Nonomura N. Mutational landscape and environmental effects in bladder cancer. Int $\mathrm{J}$ Mol Sci. 2020;21(17):E6072.

[52] Ostrander EA, Wayne RK, Freedman AH, Davis BW. Demographic history, selection and functional diversity of the canine genome. Nat Rev Genet. 2017;18(12):705-20.

[53] Ostrander EA, Dreger DL, Evans JM. Canine cancer genomics: lessons for canine and human health. Annu Rev Anim Biosci. 2019;7:449-72.

[54] LeBlanc AK, Mazcko CN. Improving human cancer therapy through the evaluation of pet dogs. Nat Rev Cancer. 2020 Sep 15. [cited 2020 Nov 11] doi: 10.1038/s41568020-0297-3. Online ahead of print. 